\title{
Can ICF model for patients with breast-cancer-related lymphedema predict quality of life?
}

\author{
Jau-Yih Tsauo • Hsiu-Chuan Hung • Han-Ju Tsai • \\ Chiun-Sheng Huang
}

Received: 13 August 2009/Accepted: 25 February 2010

(C) Springer-Verlag 2010

\begin{abstract}
Goal of work The aim of the study was to investigate if the International Classification of Functioning, Disability and Health (ICF) model with clinical data from patients with breast-cancer-related lymphedema can predict their health-related quality of life (HRQL).

Materials and methods Sixty-one patients with breastcancer-related lymphedema were recruited. Data were collected from records, including age, type(s) of surgery, number of dissected lymph nodes and history of radiotherapy and/or chemotherapy, duration of lymphedema, and duration between surgery and enrollment. Excessive arm volume, average arm symptom, function of upper extremity (U/E), and HRQL were assessed four times during and after patients' treatment of lymphedema.

Results The ICF model accounted for $20.5 \%$ to $55.6 \%$ variance in each domain of HRQL. Activity and participation reflected by U/E function were the most important factor, significantly predicting every domain of HRQL. Among measured impairments, average arm symptom was found to be most correlated with $\mathrm{U} / \mathrm{E}$ function $(r=0.590, P<0.05)$.

Conclusion The ICF model consisting of clinical measures for patients with breast-cancer-related lymphedema can predict their HRQL. Activity and participation were the
\end{abstract}

\footnotetext{
J.-Y. Tsauo $\cdot$ H.-C. Hung $\cdot$ H.-J. Tsai

School and Graduate Institute of Physical Therapy,

College of Medicine, National Taiwan University,

Taipei, Taiwan

C.-S. Huang $(\bowtie)$

Department of Surgery, College of Medicine,

National Taiwan University and Hospital,

No. 7, Chun-Shan S. Rd.,

Taipei, Taiwan

e-mail: huangcs@ntu.edu.tw
}

most important component. Arm symptoms rather than arm volume significantly correlated with U/E function. This might suggest that reducing arm symptoms is relatively more important while treating patients with breast-cancer-related lymphedema.

Keywords Breast cancer - Lymphedema - Impairment . Disability $\cdot$ Quality of life

\section{Introduction}

The cancer registry system in Taiwan (population, 23 million) documents more than 6,000 new cases of breast cancer every year. Treatments for breast cancer such as surgery and radiotherapy cause damage to lymphatic system that can result in clinically apparent lymphedema: About $28 \%$ of patients postoperatively develop lymphedema [23]. Physical, psychological, and functional aspects of life of these patients are affected [7, 25, 26, 33]. Among all of the impairments expected to negatively affect a patient's life, the question arises of which influences quality of life most.

Clinically, lymphedema is documented by limb volume $[1,2,14-16,20,21,29,31,32,34,36]$, severity of fibrosis $[4,6,18,31]$, body water composition [4], symptoms related to lymphedema $[1,3,11,36]$, and upper extremity function $[4,31]$. Limb volume is the most popularly used parameter. It is often measured by $\operatorname{limb}$ circumference [15] or water displacement [20, 21, 29, 31, 34]. Both interand intra-rater reliability for these two methods are high $[5,34,35]$. Arm symptoms reflect a patient's subjective feelings. The most common arm sensations are described as tight, heavy, painful, hard, sore, uncomfortable, hot, tumid, tingling, weak, and numb. These symptoms have 
good content validity from previous studies $[1,3,11,36]$. Moreover, Mondry et al. [22] used a $100-\mathrm{mm}$ line to present the severity of the symptoms. With compensation and help of the non-operated limb, swelling and symptoms still hamper upper extremity function; thus, inconvenience in activities of daily living continue to affect quality of life. Disability of the Arm Shoulder and Hand Outcome Questionnaire (DASH), a region-specific measure, developed to measure the upper extremity function, also shows good validity and reliability [17]. There are 30 items in the questionnaire: 21 items asking about the subjects ability to perform different tasks and nine items relating to symptoms. It uses a five-point scale with score 1 representing no difficulty or no symptom. All of these measures involve information that is clinically important in caring for patients with breast-cancer-related lymphedema.

Physical therapists' documentation was based for decades on the Nagi model of disablement [24] and WHO's International Classification of Impairments, Disabilities, and Handicaps (ICIDH) model [37]. Based on criticisms of the ICIDH framework [12], WHO released a revised version in 2001 called the International Classification of Functioning, Disability and Health (ICF) model [38]. The ICF model provides a universal language for physical therapists in research and clinical practice. It consists of the core components of physical therapy practice, functioning, activities, and participation.

It is unclear to what extent the previously mentioned impairments shown in patients with breast-cancer-related lymphedema affect the function of the upper extremity (U/E) and health-related quality of life (HRQL). We hypothesized that such clinical data do satisfy the ICF model. This study was designed to investigate if the ICF model with clinical data from patients with breast-cancer-related lymphedema can predict their HRQL.

\section{Patients and methods}

The study was conducted prospectively from August 2004 to July 2006. Before commencement of the study, the study protocol was approved by the ethics committee of our hospital. A total of 61 patients with breast-cancer-related lymphedema for at least 3 months were enrolled. Patients who had undergone bilateral mastectomy, had metastases, or had other systemic disease (such as cerebrovascular disease, renal disease, or heart disease) that could cause edema were excluded. All subjects were informed about the study purpose and signed a written consent. Through the whole treatment of lymphedema and follow-up period, everyone underwent four consecutive evaluations. Therefore, we acquired 244 evaluation data sets for analysis from the 61 patients.
Basic data, including age, body mass index (BMI) (personal factors in ICF model), dominant side, surgery type(s), number of dissected lymph nodes, and history of radiotherapy and/or chemotherapy (environmental factors in the ICF model), were collected from hospital records.

We assessed arm volume by water displacement. Both arms were measured, and excessive arm volume was defined as the difference between the operated and non-operated side. The larger the excess volume, the more severe was the visible arm swelling.

A total of 11 arm symptoms (described as tight, heavy, painful, hard, sore, uncomfortable, hot, tumid, tingling, weak, and numb) were assessed by a visual analog scale (VAS). A higher VAS score reflected a more severe symptom. Because symptoms were highly correlated with each other, we used the average VAS of all 11 symptoms for analysis of correlation and regression. Excess arm volume and average arm symptom were assigned in the body functions and structures component of the ICF model.

Function of the U/E, reflected as activity and participation component, was assessed with the DASH questionnaire, Chinese translation. The original score was transformed to a score ranging from 0 to 100 . A higher score indicated more severe dysfunction.

HRQL was assessed by the European Organization for Research and Treatment (EORTC) QLQ-C30 and EORTC QLQ-BR23 questionnaires. Fifty-three questions were aggregated, including one global HRQL measure, nine functional scales, and 13 symptom scales. For this paper, we only reported the global HRQL measure and seven functional scales. That was because we already had $11 \mathrm{arm}$ symptoms related to lymphedema. Moreover, the symptom scales in these two questionnaires were more related to the breast cancer, not so closely related to lymphedema. Two functional scales, sexual functioning and sexual enjoyment, were also omitted in this report because there was too much missing data. The absence of such data may be due to the conservative culture in this country. A higher score indicated a better status in global health status and functional scales.

All data were processed and analyzed by SPSS version 11.0 (SPSS, Chicago, IL). ${ }^{1}$ The Shapiro-Wilk test was used to examine normality of all variables. Descriptive statistics was used to present baseline data. Correlations among measures in each component (body functions and structures, environmental factors, personal factors, and activities and participation) of the ICF model were assessed using Spearman's correlation test or Pearson's correlation test. Multiple linear regression analysis was used to find the predictors of HRQL in the ICF model. To determine factors affecting each domain of HRQL

\footnotetext{
${ }^{1}$ SPSS version 11.0 (SPSS, Chicago, IL, USA).
} 
(EROTC QLQ C-30 and EROTC QLQ BR-23), univariate analysis or bivariate correlation was first carried out. Significant predictors were then used as independent variables in the further stepwise multiple regressions. The potential independent variables included age, BMI, surgical type, CT, RT, number of lymph node dissected, lymphedema on dominanat hand, average arm symptom, excessive arm volume, and DASH score. Square of the correlation coefficient $\left(r^{2}\right)$ is the coefficient of determination, which represented the percentage of the total variance in the scales of dependent variables, and can be explained by the independent variables. The $\alpha$ level was set at 0.05 .

\section{Results}

Mean age of the 61 patients was 54.0 years $(\mathrm{SD}=8.9$, range $=$ 36-75). Their BMI was $24.7 \mathrm{~kg} / \mathrm{m}^{2}(\mathrm{SD}=3.8$, range $=17.9$ 40.3). Types of surgery and treatment characteristics are summarized in Table 1. Descriptive data regarding arm symptoms, arm excess volume, and DASH score are summarized in Table 2. We found that upper extremity dysfunction was not prominent (score 15.7 \pm 13.0 ). Tightness and tumidness were the most severe $(\mathrm{VAS}=3.7)$ and most frequent (over 90\%) symptoms among all measured. Functional scale data from the QLQ C-30 and QLQ BR-23 are summarized in Table 3. Global health status $(66.9 \pm 21.7)$, body image $(78.4 \pm 21.0)$, and future perspective $(56.4 \pm 29.7)$ were the three worst scales.

We developed an ICF model for patients with breastcancer-related lymphedema. Correlations among measures in each component of the ICF model were investigated and were listed in Table 4. Average arm symptom was significantly correlated with history of chemotherapy $(r=0.207, P<0.05)$. Excessive arm volume was significantly correlated with age

Table 1 The surgery types and treatment characteristics $(N=61)$

\begin{tabular}{llr}
\hline & Number & Percentage \\
\hline Surgery types & & \\
$\quad$ Radical mastectomy & 2 & 3.3 \\
$\quad$ Modified radical mastectomy & 50 & 82.0 \\
Simple mastectomy & 4 & 6.6 \\
$\quad$ Breast conservation surgery & 5 & 8.2 \\
Radiotherapy & 46 & 75.4 \\
Chemotherapy & 55 & 90.2 \\
Lymphedema on dominant hand & 36 & 59.0 \\
$\begin{array}{l}\text { Number of lymph node dissected } \\
\text { (mean (SD)) }\end{array}$ & 18.9 (9.0) & \\
$\begin{array}{l}\text { Duration of lymphedema } \\
\text { (month, mean (SD)) }\end{array}$ & 33.7 (39.7) & \\
$\begin{array}{l}\text { Duration between surgery and } \\
\text { enrollment (month, mean (SD)) }\end{array}$ & $61.4(54.8)$ & \\
\hline
\end{tabular}

Table 2 The descriptive data of arm symptoms, excessive arm volume, and function $(N=244)$

\begin{tabular}{lrrc}
\hline & Mean (SD) & Median & $\begin{array}{l}\text { \% of patient } \\
\text { with the } \\
\text { symptom }\end{array}$ \\
& & & \\
\hline Arm symptoms (VAS) & $3.7(2.8)$ & 3.2 & 94.3 \\
Tight & $3.2(2.8)$ & 2.3 & 87.3 \\
Heavy & $1.7(2.4)$ & 0.5 & 61.9 \\
Pain & $3.1(2.9)$ & 2.2 & 88.1 \\
Hard & $2.7(2.9)$ & 1.6 & 76.6 \\
Sore & $3.1(3.0)$ & 2.1 & 87.3 \\
Uncomfortable & $1.7(2.4)$ & 0.5 & 58.2 \\
Hot & $3.7(3.1)$ & 2.8 & 91.8 \\
Tumid & $1.6(2.4)$ & 0.2 & 54.9 \\
Tingle & $2.5(3.0)$ & 0.9 & 64.3 \\
Weak & $2.3(3.0)$ & 0.8 & 65.6 \\
Numb & $2.7(2.4)$ & 1.8 & \\
Average arm symptom & $466.2(318.6)$ & 361.3 & \\
Excessive arm volume (ml) & $15.7(13.0)$ & 13.3 & \\
Function (DASH) & & \\
\hline
\end{tabular}

$V A S$ visual analog scale, DASH Disability of the Arm Shoulder and Hand Outcome Questionnaire

( $r=0.325, P<0.05)$, BMI $(r=0.393, P \leq 0.05)$, and history of chemotherapy $(r=0.167, P<0.05)$. DASH functional score was significantly correlated with age $(r=0.140, P<0.05)$, history of chemotherapy $(r=0.149, P<0.05)$, and average arm symptom $(r=0.590, P<0.05)$.

Stepwise multiple linear regression was used to predict domains of HRQL (EROTC QLQ C-30 and EROTC QLQ BR-23) with measures in each component in the ICF model, and results were shown in Table 5. DASH functional score was the most important predictor, significantly influencing every domain of HRQL. Physical functional domain and role functional domain were most predictable with only one or two variables, and the $R^{2}$ values were 0.556 and 0.539 , respectively. All domains

Table 3 The descriptive data of functional scales in EORTC QLQ-C30 and QLQ-BR23 questionnaires $(N=244)$

\begin{tabular}{llr}
\hline Scales of HRQL & Mean (SD) & Median \\
\hline Global health status & $66.9(21.7)$ & 66.7 \\
Physical functional & $85.4(13.1)$ & 86.7 \\
Role functional & $82.6(21.1)$ & 100.0 \\
Emotional functional & $80.4(21.1)$ & 83.3 \\
Cognitive functional & $81.4(19.1)$ & 83.3 \\
Social functional & $82.1(22.1)$ & 83.3 \\
Body image & $78.4(21.0)$ & 83.3 \\
Future perspective & $56.4(29.7)$ & 66.7 \\
\hline
\end{tabular}


Table 4 Correlations among measures in each component (body functions and structures, environmental factors, personal factors, activities, and participation) of ICF model

\begin{tabular}{llll}
\hline & $\begin{array}{c}\text { Average } \\
\text { symptom }\end{array}$ & $\begin{array}{l}\text { Excessive } \\
\text { arm volume }\end{array}$ & DASH \\
\hline $\begin{array}{l}\text { Personal factors } \\
\text { Age }\end{array}$ & 0.022 & $0.325^{*}$ & $0.140^{*}$ \\
BMI $^{\mathrm{b}}$ & 0.007 & $0.393^{*}$ & -0.123 \\
Environmental factors $^{\text {Surgical type }}$ & & & \\
Radiotherapy $^{\mathrm{b}}$ & 0.054 & -0.084 & 0.051 \\
Chemotherapy $^{\mathrm{b}}$ & 0.085 & 0.047 & 0.113 \\
Number $^{\mathrm{a}}$ & $0.207^{*}$ & $0.167^{*}$ & $0.149^{*}$ \\
Body functions and structures $^{\text {Side }}$ & 0.030 & -0.020 & -0.032 \\
Average symptom $^{\mathrm{b}}$ & -0.062 & 0.094 & -0.003 \\
Excessive arm volume $^{\mathrm{a}}$ & & 0.034 & $0.590^{*}$ \\
\hline
\end{tabular}

Surgical type types of surgery underwent, Number number of lymph node dissected, Side if lymphedema on dominant hand, Symptom average of arm symptoms in visual analog scale, DASH Disability of the Arm Shoulder and Hand Outcome Questionnaire, functional score

$* P<0.05$

${ }^{a}$ Pearson's correlation

${ }^{\mathrm{b}}$ Spearman's correlation

could be predicted by components in the ICF model with $R^{2}$ values ranging from 0.205 (body image domain) to 0.556 (physical functional domain).

\section{Discussion}

The results of this study indicate that an ICF model constructed with clinical measures used by physical therapists can readily explain the HRQL of patients with breast-cancerrelated lymphedema. Results of multiple regression analysis showed that clinical measures accounted for $20.5 \%$ to $55.6 \%$ in each domain of HRQL (Table 5). In the study by Pierce et al. [27], they found the combination of ICF components only accounted for $17 \%$ of life satisfaction among patients with traumatic brain injury. It seems that the life satisfaction of this patient population was more unpredictable than that of our patients.

Among the components of the ICF model, activity and participation (DASH score in this study) were the most important factor, significantly predicting every domain of HRQL, especially the physical functional and role functional domains. Our findings are in line with those of others. Pierce et al. [27] also found that participation was the strongest predictor. Hack et al. [10] found that symptoms and associated disabilities were significant predictors of quality of life among patients. According to Engel's 5-year prospective study in breast cancer patients, quality of life was significantly lower $(P<0.001)$ for patients with arm difficulties [8]. However, in Rietman's study [28], they did not consider disability a predictor. Instead, they found that the physical functioning domain in HRQL could be predicted by age and impairments (e.g., shoulder flexion limitation, grip strength, and arm volume) and accounted for $52 \%$ of variance. In our study, age as the personal factor, surgical type in environmental factors, and average arm symptom in body functions and structures each significantly predicted four domains (Table 5).

Which impairment best correlated with the function of our patients? Average arm symptom was found to be the most tightly correlated. Clinically, physical therapists put much emphasis on reducing swelling of the arms, but perhaps we should shift attention to improving arm symptoms, not just circumference or volume. Although many of the arm symptoms are closely related to arm volume, in Rietman's study [28], they showed that disability was predicted mostly by one arm symptom and pain (61\%). Arm swelling did not have a significant correlation with function. Segerström et al. [30] also found rather low relative risks between the presence of arm edema and estimated disabilities. That finding also suggests that physicians should not decide a patient's need of medical help only according to arm volume.

Rather than use the measured symptom of pain as most studies have done, we measured 11 related symptoms and derived an average symptom score. We found that only history
Table 5 Results of multiple linear regression for the predictors of domains of HRQL (EROTC QLQ C-30 and EROTC QLQ BR-23)

$r^{2}$ square of the correlation coefficient, the coefficient of determination, $D A S H$ Disability of the Arm Shoulder and Hand Outcome Questionnaire, $C T$ chemotherapy, $R T$ radiotherapy, $B M I$ body mass index

\begin{tabular}{lll}
\hline Scales of HRQL & Predictors & $r^{2}$ \\
\hline Global health status & DASH, CT, average arm symptom, excessive arm volume, surgical type & 0.297 \\
Physical functional & DASH, age & 0.556 \\
Role functional & DASH & 0.539 \\
Emotional functional & DASH, average arm symptom, surgical type, RT & 0.463 \\
Cognitive functional & DASH, RT, excessive arm volume, age & 0.343 \\
Social functional & DASH, surgical type, BMI & 0.381 \\
Body image & DASH, age, average arm symptom & 0.205 \\
Future perspective & DASH, age, BMI, average arm symptom, surgical type & 0.362 \\
\hline
\end{tabular}


of chemotherapy in environmental factors significantly correlated with average symptom and function. Hack et al. [10] also found that pain severity correlated positively with history of chemotherapy but not radiotherapy.

In this study, age, BMI, and if having received chemotherapy or not were significantly correlated with excessive arm volume, but types of surgery, history of radiotherapy, or dosage of radiotherapy, and number of lymph nodes dissected were not correlated with excessive arm volume. Opposite to our result, types of surgery that influenced the edema and limb function was reported in literatures [9, 23]. Most of our subjects $(82.0 \%)$ underwent modified radical mastectomy. Maybe this uneven distribution caused the result of no significant correlation between different operation procedures and the symptoms and excessive arm volume. Kärki et al. found no significant difference between patients with different postoperative adjuvant treatments (chemotherapy, radiotherapy, and hormonal therapy) in the incidence of impairments [13]. Gosselink et al. also found there was no effect of axillary irradiation on arm circumference [9]. However, Motimer et al. [23] found that the prevalence of lymphedema of subjects receiving radiotherapy was 2.45 times of that of subjects without receiving radiotherapy. Maunsell et al. [19] concluded that edema of arm was correlated significantly with receiving radiotherapy and axillary lymph node dissection. There might be different bases in comparisons among these research findings with ours. Some $[13,19,23]$ investigated the prevalence of lymphedema and impairments, and other researchers, including us, investigated the severity among subjects already having lymphedema. Of course, recent sentinel node procedure and progression in the treatment regimen might cause the different pictures of residual effects of treatment.

There are limitations in this study, which should be mentioned. First of all, although there were 244 evaluation data in this study, they were from only 61 patients. The small sample size might make the generalization more limited. Secondly, there were no data of the occupation of these patients, which might be one of the factors affected their quality of life. And, the detailed data of treatment protocol, such as the chemotherapy protocol and radiotherapy protocol, were lacking. These factors might also influence the patients' impairments, function, and quality of life.

In conclusion, our study indicates that an ICF model constructed with clinical measures for patients with breastcancer-related lymphedema can predict their HRQL with $20.5 \%$ to $55.6 \%$ variance in each domain. Activity and participation were the most important component to predict HRQL. Arm symptoms rather than arm volume significantly correlated with upper extremity function. This conclusion implies that it might be more important to reduce arm symptoms than arm volume while treating patients with breast-cancer-related lymphedema to improve both upper extremity function and HRQL.

Acknowledgments The authors thank the participants and the National Science Council of the Republic of China for financial support under grant nos. NSC93-2314-B-002-118 and NSC94-2314B-002-074, which made this study possible.

\section{References}

1. Andersen L, Hojris I, Erlandsen M, Andersen J (2000) Treatment of breast-cancer-related lymphedema with or without manual lymphatic drainage — a randomized study. Acta Oncol 39:399-405

2. Bunce IH, Miroli BR, Hennessy JM, Ward LC, Jones LC (1994) Postmasectomy lymphoedema treatment and measurement. Med J Aust 161:125-128

3. Caban ME (2002) Trends in the evaluation of lymphedema. Lymphology 35:28-38

4. Carati CJ, Anderson SN, Gannon BJ, Piller NB (2003) Treatment of postmasecotmy lymphoedema with low level laser therapy. Cancer 98:1114-1122

5. Chen YW, Tsai HJ, Hung HC, Tsauo JY (2008) Reliability study of measurements for lymphedema in breast cancer patients. Am J Phys Med Rehabil 87:33-38

6. Clodius L, Deak L, Piller NB (1976) A new instrument for the evaluation of tissue tonicity in lymphoedema. Lymphology 9:1-5

7. Cohen SR, Payne DK, Tunkel RS (2001) Lymphedema. Cancer 92:980-987

8. Engel J, Kerr J, Schlesinger-Raab A, Sauer H, Holzel D (2003) Axilla surgery severely affects quality of life: results of a 5-year prospective study in breast cancer patients. [Erratum appears in Breast Cancer Res Treat 2003 Jul;80(2):233]. Breast Cancer Res Treat 79:47-57

9. Gosselink R, Rouffaer L, Vanhelden P, Piot W, Troosters T, Christiaens MR (2003) Recover of upper limb function after axillary dissection. J Surg Oncol 83:204-211

10. Hack TF, Cohen L, Katz J, Robson LS, Goss P (1999) Physical and psychological morbidity after axillary lymph node dissection for breast cancer. J Clin Oncol 17:143-149

11. Hladiuk M, Huchcroft S, Temple W, Schnurr BE (1992) Arm function after axillary dissection for breast cancer: a pilot study to provide parameter estimates. J Surg Oncol 50:47-52

12. Jette AM (2006) Toward a common language for function, disability, and health. Phys Ther 86:726-734

13. Kärki A, Simonen R, Mälkiä E, Selfe J (2005) Impairments, activity limitations and participation restrictions 6 and 12 months after breast cancer operation. J Rehabil Med 37:180-188

14. Ko DSC, Lerner R, Klose G, Cosimi AB (1998) Effective treatment of lymphoedema of the extremities. Arch Surg $133: 452-458$

15. Leduc O, Leduc A, Bourgeois P, Belgrado JP (1998) The physical treatment of upper limb edema. Cancer 83(12 Suppl American):2835-2839

16. Liao SF, Huang MS, Li SH, Chen IR, Wei TS, Kuo SJ, Chen ST, Hsu JC (2004) Complex decongestive physiotherapy for patients with chronic cancer-associated lymphedema. J Formos Med Assoc 103:344-348

17. Liang HW, Wang HK, Yao G, Horng YS, Hou SM (2004) Psychometric evaluation of the Taiwan version of the disability of the arm, shoulder, and hand (DASH) questionnaire. J Formos Med Assoc 103:773-779

18. Liu NF, Olszewski W (1992) Use of tonometry to assess lower extremity lymphedema. Lymphology 25:155-158 
19. Maunsell E, Brisson J, Deschènes L (1992) Arm problems and psychological distress after surgery for breast cancer. Can J Surg 36:315-320

20. McKenzie DC, Kalda AL (2003) Effect of upper extremity exercise on secondary lymphedema in breast cancer patients: a pilot study. J Clin Oncol 21:463-466

21. Megens AM, Harris SR, Kim-Sing C, McKenzie DC (2001) Measurement of upper extremity volume in women after axillary dissection for breast cancer. Arch Phys Med Rehabil 82:16391644

22. Mondry TE, Riffenburgh RH, Johnstone PA (2004) Prospective trial of complete decongestive therapy for upper extremity lymphedema after breast cancer therapy. Cancer J 10:42-48

23. Motimer PS, Bates DO, Brassington HD, Stanton AWB, Strachan DP, Levick JR (1996) The prevalence of arm oedema following treatment for breast cancer. Q J Med 89:377-380

24. Nagi SZ (1964) A study in the evaluation of disability and rehabilitation potential: concepts, methods, and procedures. Am J Public Health Nations Health 54:1568-1579

25. Pain SJ, Purushotham AD (2000) Lymphoedema following surgery for breast cancer. Br J Surg 87:1128-1141

26. Passik SD, McDonald MV (1998) Psychosocial aspects of upper extremity lymphedema in women treated for breast carcinoma. Cancer 83(12 Suppl American):2817-2820

27. Pierce CA, Hanks RA (2006) Life satisfaction after traumatic brain injury and the World Health Organization model of disability. Am J Phys Med Rehabil 85:889-898

28. Rietman JS, Dijkstra PU, Debreczeni R, Geertzen JHB, Robinson DPH, De Vries J (2004) Impairments, disabilities and health related quality of life after treatment for breast cancer: a follow-up study 2.7 years after surgery. Disabil Rehabil 26:78-84
29. Sander AP, Hajer NM, Hemenway K, Miller AC (2002) Upperextremity volume measurements in women with lymphedema: a comparison of measurements obtained via water displacement with geometrically determined volume. Phys Ther 82:1201-1212

30. Segerström K, Bjerle P, Nystrom A (1991) Importance of time in assessing arm and hand function after treatment of breast cancer. Scand J Plast Reconstr Surg Hand Surg 25:241-244

31. Szuba A, Achalu R, Rockson SG (2002) Decongestive lymphatic therapy for patients with breast carcinoma-associated lymphedema. A randomized, prospective study of a role for adjunctive intermittent pneumatic compression. Cancer 95:2260-2267

32. Szuba A, Cooke JP, Yousuf S, Rockson SG (2000) Decongestive lymphatic therapy for patients with cancer-related or primary lymphedema. Am J Med 109:296-300

33. Tobin MB, Lacey HJ, Meyer L, Mortimer PS (1993) The psychological morbidity of breast cancer-related arm swelling. Psychological morbidity of lymphoedema. Cancer 72:3248-3252

34. Taylor R, Jayasinghe UW, Koelmeyer L, Ung O, Boyages J (2006) Reliability and validity of arm volume measurements for assessment of lymphedema. Phys Ther 86:205-214

35. Tsai HJ, Liu YX, Tsauo JY (2005) Reliability study of lymphedema measurement. Formos J Phys Ther 30:124-131

36. Williams AF, Vadgama A, Franks PJ, Mortimer PS (2002) A randomized controlled crossover study of manual lymphatic drainage therapy in women with breast cancer-related lymphoedema. Eur J Cancer Care 11:254-261

37. World Health Organization (1980) International classification of impairments, disabilities, and handicaps: a manual of classification relating to the consequences of disease. World Health Organization, Geneva

38. World Health Organization (2001) ICF-International classification of functioning, disability and health. WHO Library, Geneva 